\title{
Hacia un concepto riguroso de la experiencia del dolor: la fenomenología, su método y sus consecuencias
}

\author{
Francisco Mujica \\ Université catholique de Louvain-La-Neuve, Bélgica
}

\begin{abstract}
ResUmeN: La filosofía fenomenológica nace como respuesta a las limitaciones descriptivas y epistemológicas de las ciencias parciales. En tanto las ciencias parciales renuncian a reflexionar sobre sus presupuestos en nombre de su operatividad, ellas resultan en una descripción intelectualizada del objeto y/o del ámbito experiencial descritos. En este sentido, una fenomenología del dolor humano se revela como una contribución capital para sobreponerse a las inconsistencias analíticas del enfoque científico del estudio del dolor (paradigma biomédico). A partir de una reconstrucción de los presupuestos fundamentales del enfoque científico del estudio del dolor (1), este artículo mostrará las debilidades epistemológicas y las insuficiencias descriptivas del enfoque científico del estudio del dolor (2). A continuación se procederá a establecer los principios epistemológicos y metodológicos
\end{abstract}

\footnotetext{
Francisco Musica es sociólogo de la Pontificia Universidad Católica de Chile y Doctor en Filosofía, Université catholique de Louvain-La-Neuve, Bélgica. Investigador visitante del Institut Supérieur de Philosophie, Université catholique de Louvain-La-Neuve. Dirección: Julio Montebruno 651, La Reina, Santiago, Chile, CP 7850000. Email: franciscomujica82@gmail.com.

Este artículo es fruto de mi investigación sobre fenomenología del dolor como estudiante de doctorado en Filosofía de la Universidad de Lovaina (2014-2018). Agradezco al Institut Supérieur de Philosophie de la Universidad de Lovaina por extenderme una invitación a permanecer como investigador visitante en el año académico 2018-2019, período durante el cual pude profundizar en algunos conceptos expuestos en este artículo. Agradezco, asimismo, a los evaluadores anónimos de este artículo por sus comentarios; algunos de ellos resultaron vitales para una mejor exposición del argumento. Todos los errores y omisiones son de mi exclusiva responsabilidad.
} 
diferenciales de la filosofía fenomenológica (regreso a la experiencia originaria, intencionalidad/pasividad de la consciencia), así como la pertinencia de estos a la hora de abordar el fenómeno del dolor (3). Las herramientas diferenciales de la fenomenología nos permitirán llevar a cabo un esbozo de descripción fenomenológica del dolor (4). Concluiremos este artículo con una reflexión sobre las consecuencias programáticas y terapéuticas de contar con un concepto riguroso de la experiencia del dolor humano - concepto específicamente posibilitado por la descripción fenomenológica (5).

Palabras Clave: fenomenología, dolor, enfoque científico del estudio del dolor, experiencia, límite, cuerpo vivido, alienación, autoidentificación RECIBIDO: febrero 2020 / ACEPTADO: junio 2020

\section{Towards a Rigorous Concept of the Experience of Pain: Phenomenology, Its Method and Consequences}

ABSTRACT: Phenomenology arises as a critique of epistemological shortage in positive sciences. Given that positive sciences refuse to carry out a critical review of their own intellectual assumptions, positive sciences eventually deliver an intellectualized account of experience. In this way, a phenomenological description of human pain reveals itself as a major contribution to overcoming the analytical shortage of classical approaches to pain (namely naturalistic). First of all, we will outline the main features of classical approaches to pain (1), with the intention of underlining the epistemological weakness, as well as the poor descriptive outcome of naturalistic approaches to pain (2). That being said, we will establish the epistemological and methodological principles of phenomenology - 'things themselves', intentionality/ passivity of consciousness - as well as their relevance to grasping human pain (3). Moreover, these principles will allow us to sketch a phenomenological description of pain experience (4). We will conclude with a brief reflection on the programmatic and the therapeutic consequences of having a rigorous concept of pain experience - especially considering that a rigorous concept of pain experience is the ultimate outcome of a phenomenological description (5).

KEYWORDS: phenomenology, pain, naturalistic approach to pain, experience, limit, lived-body, estrangement, self-resonance

ReCeived: February 2020 / AcCePted: June 2020 


\section{La fenomenología y el esquema científico como paradigma dominante del estudio del dolor}

a fenomenología es un movimiento filosófico iniciado en Alemania

L por E. Husserl y que, a lo largo del siglo XX, ha dado grandes pensadores como M. Heidegger, M. Scheler, E. Lévinas o E. Stein (Spiegelberg 1994). Como toda filosofía, la fenomenología es una tentativa de captar la integralidad diferencial o la consistencia privativa del objeto referido o de la experiencia realizada. No obstante, la especificidad de la fenomenología se nos revela con total nitidez cuando se explicita la particularidad de su método (Lévinas 1968). Si el gesto de captura filosófica en el empirismo es la inducción de una generalidad a partir de relaciones de probabilidad entre manifestaciones contingentes $y$, en el caso del idealismo, la subsunción del objeto para posicionarlo en un marco idealmente construido —en un esquema- (Ortega y Gasset 1975), la fenomenología procede de una forma radicalmente diferente.

La unicidad del método fenomenológico reside en que él pretende alcanzar la integralidad de lo experimentado a partir de la pura descripción de la donación del objeto vivenciado, es decir, al margen de toda hipótesis preestablecida y con independencia de toda atribución judicativa: "La fenomenología es la ciencia de los fenómenos. Esto significa que ella es una descripción anterior a toda teoría e independiente de toda presuposición" (Henry 2011, 59; mi traducción).

Podemos ver, entonces, que la pretensión de la fenomenología no es nada fácil. No solamente por el desafío epistemológico que implica toda descripción esencial de la pura donación, sino porque la fenomenología enfrenta un obstáculo disciplinario resultante del derrotero sociohistórico de las ciencias positivas (física, química, biología, etcétera). Husserl $(1990, \S 9)$ observa que la cultura occidental —nuestro 'mundo de la vida' (Lebenswelt) - ha sufrido una intelectualización como consecuencia de la introducción incontrolada de las ciencias positivas como mecanismo dominante de descripción del hombre, la sociedad, la historia y la cultura. Ejemplos de esto son la utilización del mecanicismo como estrategia descriptiva de la experiencia del cuerpo humano, la apropiación irreflexiva de los presupuestos de la física mecánica en la codificación iuspositivista del derecho o la importación incuestionada del cientificismo en psicología con el proyecto decimonónico de una 'psicología sin alma' (Boutot 1995). Husserl (1990, §38) descubre que nuestra 
época es la primera en donde "un mundo objetivado oculta al mundo que se vivencia efectivamente". Evidentemente que la experiencia del dolor humano no escapa a esta cientifización no autocomprensiva propia de nuestro tiempo.

En este sentido, una fenomenología del dolor no solo deberá llevar a cabo una descripción esencial del fenómeno del dolor, sino que, más aún, deberá hacerse cargo del estado del arte en el que la experiencia del dolor se encuentra como consecuencia de la problemática caracterización de este por parte de las ciencias parciales. Dado lo anterior, el argumento fundamental de este artículo es el siguiente. En primer lugar, mostraremos cómo las ciencias parciales han descrito el dolor, cuál es el estado del arte y las insuficiencias epistemológicas que una descripción fundamental de la experiencia del dolor debe enfrentar. A continuación se explicitarán las herramientas diferenciales que permiten a la fenomenología llevar a cabo un intento de descripción esencial del fenómeno del dolor. Finalmente, se subrayarán las prestaciones (teóricas y prácticas) de contar con una descripción rigurosa de la experiencia del dolor humano.

Veamos, entonces, cuál es el enfoque dominante con el que las ciencias parciales han pretendido describir el fenómeno del dolor.

No cabe duda de que el esquema analítico dominante del estudio del dolor en nuestra época es el enfoque científico del dolor. Desde el punto de vista científico, el dolor es concebido como una consecuencia de la estructura anatómica que da lugar a la sensación. De esta forma, el dolor es necesariamente tributario del funcionamiento del sistema nervioso central: el dolor es, así, un tipo de sensación activada por un estímulo o por una causa externa al sistema nervioso central (Purves 1997). Entender el dolor en el esquema científico implica forzosamente comprender la estructura de la sensación (Kandel, Schwartz y Jessell 2001).

La investigación biomédica contemporánea procede a enfocar la sensación desde una perspectiva operacional. Es así que el esquema científico de comprensión del dolor accede a su estudio a partir de la delimitación de funciones sensitivas diferenciales, a saber: la codificación de estímulos exteriores (mecánicos, térmicos y/o químicos), la transformación de estos en energía y su ulterior transmisión hacia el sistema nervioso central (Purves 1997). Existen dos tipos de receptores de codificación y de transmisión de influjos sensitivos: mecanosensitivos (espe- 
cializados en la detección del movimiento) y termonociceptivos (especializados en la detección de temperatura y dolor) (Kandel, Schwartz y Jessell 2001).

A pesar de que el desencadenamiento de las sensaciones motora y termonociceptiva comparte el modo de activación (estímulo que deforma las terminaciones nerviosas induciendo una sinapsis en las células receptoras), los dos tipos de sensorreceptores presentan diferencias solo comprensibles en virtud de una profunda especialización de sus funciones respectivas. Si por un lado los mecanorreceptores tienen un umbral de activación bajo y una capacidad de adaptación alta, los receptores termonociceptivos tienen un umbral de activación alto y una capacidad de adaptación baja (Merchand 2009).

Asimismo, debe destacarse una diferencia fundamental entre los receptores mecanosensitivos y termonociceptivos en la operatividad de la sensibilidad. Mientras el desencadenamiento de la sensación en el caso del estímulo mecanosensitivo depende de la activación de diversos mecanorreceptores cutáneos distribuidos en la superficie corporal y en los tejidos musculares, la sensación dolorosa o de calor es gatillada por receptores termonociceptivos que se encuentran presentes en toda la piel. Es precisamente por esto que el dolor comparte en general su tenor estesiológico con el de las quemaduras (Purves 1997).

Al margen de que el enfoque científico establece que la sensación es una suerte de precondición del dolor, este último no puede entenderse como una mera hiperestimulación de los receptores sensitivos. La razón es simple: la estructura y la funcionalidad anatómicas de la sensación dolorosa se distingue de la mera sensación en virtud de la autonomía y diferenciación de diversas operaciones orgánicas (Merchand 2009).

Un primer indicador de la distinción recién señalada es la que realiza el sistema nervioso central entre dolor y sensibilidad: el cerebro activa una señal de alerta frente al dolor (lo que testimonia la autonomía de la nocicepción frente a la mera estimulación sensitiva). Más aún, la especificidad diferencial de la nocicepción se ratifica cuando se comprende el grado de diferenciación estructural que revela el tipo de axón que está a la base de la transmisión del dolor: la estructura de los axones del dolor es de tipo amielínica, lo que tiene como consecuencia que la transmisión del dolor sea cien veces más lenta que la de la sensación no dolorosa (Purves 1997). Por último, un punto adicional que refrenda la autonomía 
de la consistencia de la sensación dolorosa remite a la alta especialización que exhiben los receptores del dolor, a saber: los nociceptores (Merchand 2009).

La diferenciación de la consistencia anatómica de los nociceptores es otro punto en donde el dolor testimonia su autonomía funcional. En este sentido, los nociceptores son más bien lentos en relación con los sensorreceptores, aunque los primeros poseen una vía rápida y una vía lenta. De ahí que el enfoque científico del estudio del dolor descubra que en toda experiencia dolorosa se manifiesten dos dolores: el primero es atribuible a la vía rápida de transmisión (agudo y súbito, como un pinchazo), mientras que el segundo es imputable a la acción de la vía lenta de transmisión (sensación tardía, profunda y difusa, como una quemadura) (Purves 1997). La razón detrás de esta relación reside en la especialización de las vías de transmisión axonales de los nociceptores: en tanto el primer dolor depende de zonas específicas de la corteza cerebral, él determina la localización, la intensidad y la cualidad de la estimulación (Kandel, Schwartz y Jessell 2001). En contraposición, el segundo dolor da cuenta del carácter desagradable de la experiencia dolorosa, activando así la dimensión volitiva (rechazo, reacción), pues este recorrido depende de las vías corticales del tronco cerebral (Merchand 2009).

Junto con la estructura de los 'dos dolores' que deriva de la diferenciación anatómica de los nociceptores, el dolor también muestra una diferenciación cualitativa. No por casualidad podemos distinguir 'tipos' de dolor: el dolor puede 'raspar', 'arder', 'apretar', 'desgarrar', 'roer'; todas expresiones adecuadas que no le quitan al fenómeno su registro característico. El esquema científico del estudio del dolor descubre el motivo de estos matices estesiológicos en la diferenciación interna de los nociceptores, los que se dividen en tres tipos: mecanonociceptores (se activan frente a cortes y raspaduras), termonociceptores (que reaccionan al frío y al calor) y nociceptores polimodales (sensibles a movimientos bruscos, golpes secos y sustancias químicas) (Merchand 2009). Más allá de la alta especialización cualitativa de los nociceptores, todo dolor requiere de un cierto umbral —ya de presión, ya de acidez, ya de calor — para desencadenarse (Melzack y Wall 1989).

Pero el esquema científico de estudio del dolor descubre que todo dolor alcanza igualmente su condición diferencial como consecuencia 
de sus mecanismos específicos de transmisión. En ese sentido, se pueden distinguir las vías aferentes - desde la periferia hacia el sistema nervioso central a través de la médula espinal (que determina la intensidad del dolor)—, y las vías eferentes, donde se genera la sensación desagradable y la extensión temporal del dolor (desde el sistema nervioso central, pasando por la médula espinal hasta la periferia) (Merchand 2009). Sin embargo, lo más notable del sistema de transmisión del dolor remite al hecho de que cada etapa del proceso exhibe mecanismos de activación y de inhibición (Melzack y Wall 1989): toda sinapsis dolorosa cuenta con dispositivos de desencadenamiento y de bloqueo (tanto a nivel del sistema aferente como eferente) (Wall y Melzack 1999). El esquema científico del estudio del dolor revela con esto que todo dolor, no solo tiene como condición de posibilidad la sensación, sino que, asimismo, una suerte de 'deliberación' anatómica y nerviosa desde la cual el dolor surge (o desde la cual es inhibido) (Merchand 2009).

Esta estructura recién descrita también le permite al enfoque científico dar cuenta de ciertas particularidades contraintuitivas características del fenómeno del dolor, como lo es la somatización temporal: en tanto la velocidad de la captación no coincide con la de la transmisión del dolor, este puede sentirse con posterioridad a la estimulación (Merchand 2009). Algo análogo ocurre con el fenómeno de la somatización espacial: una estimulación de la misma potencia será percibida con mayor intensidad si ella tiene lugar en una superficie grande $-\mathrm{y}$ con menor intensidad en una superficie pequeña (Melzack y Wall 1989). Más aún: la somatización espacial del dolor puede manifestarse a veces en un lugar diferente del órgano afectado, fenómeno denominado por el esquema científico como 'dolor irradiado' (Purves 1997).

El dolor irradiado puede explicarse por cuatro motivos:

1. Las ramas de una vía aferente se dividen para transmitir al sistema nervioso central, por un lado, la localización y, por otro, la profundidad. Al recibir dos informaciones distintas, el cerebro asocia el dolor a una región cutánea (y no a la región intraorgánica).

2. La liberación de sustancias algógenas se produce en una zona distinta de la parte afectada (el cerebro identifica correctamente el lugar del dolor, pero no la zona estimulada). 
3. La estimulación provoca la contracción de músculo alrededor del órgano afectado (y el dolor es percibido solo en su tenor muscular).

4. La transmisión del influjo es realizado a una neurona de proyección, lo que conlleva que la reacción del sistema nervioso central atribuya el dolor a la zona proyectada (Merchand 2009).

De la misma forma que la dimensión espacial le permitió al enfoque científico revelar ciertas curiosas particularidades del fenómeno del dolor, su enfoque de la dimensión temporal entregará nuevos matices en la descripción del dolor. La investigación científica sobre el sistema nervioso central subraya su plasticidad, vale decir, su capacidad de modificarse a sí mismo a partir de sus propias operaciones. Es por esto que ciertos estímulos muy intensos y/o prolongados pueden alterar su funcionamiento. El enfoque científico encuentra en este dato la clave para aproximarse a la cuestión del dolor crónico (Merchand 2009). A diferencia del dolor provocado por una estimulación, el dolor crónico es considerado por el enfoque científico como una enfermedad, la que puede adoptar dos variantes: hipersensibilización local (alodinia, resultante de una quemadura) e hipersensibilización central (daño al sistema nervioso central) (Merchand 2009).

El análisis de los factores y variantes del dolor recientemente esbozado permite al esquema científico generar tres criterios de clasificación del dolor:

1. Duración del dolor. Si se trata de un dolor derivado directamente de una estimulación, hablamos de un dolor agudo. Por el contrario, si el dolor dura más de treinta días después de la estimulación, se hablará de dolor crónico (Purves 1997).

2. Tipo de nociceptor activado. El dolor resultante de una herida cortante no es exactamente igual al de uno derivado de una quemadura o de un accidente químico.

3. Tipo de lesión o daño. El dolor que resulta de una herida superficial cutánea no es equivalente a uno procedente de una disfunción del sistema central (Merchand 2009).

La reconstrucción de los hallazgos y presupuestos del enfoque científico del estudio del dolor nos permite ahora proponer un panorama de las variantes, causas y mecanismos del dolor (así como de sus características y respuestas típicas): 
Tabla 1. VARIANTES, CAUSAS Y MECANISMOS DEL DOLOR

\begin{tabular}{|c|c|c|c|}
\hline Tipo de dolor & & Mecanismos & Respuesta/sensación \\
\hline \multirow[t]{2}{*}{ Nociceptivo } & $\begin{array}{c}\text { Somática } \\
\text { (laceración, fractura, } \\
\text { dolor posoperatorio) }\end{array}$ & $\begin{array}{c}\text { Estimulación } \\
\text { mecánica, térmica o } \\
\text { química }\end{array}$ & $\begin{array}{c}\text { Dolor superficial o } \\
\text { profundo, reflejo } \\
\text { nociceptivo y respuesta } \\
\text { automática }\end{array}$ \\
\hline & $\begin{array}{c}\text { Visceral } \\
\text { (colitis, cistitis) }\end{array}$ & Distensión de vísceras & $\begin{array}{c}\text { Constante, mal localizado } \\
\text { (irradiado) y de respuesta } \\
\text { automática }\end{array}$ \\
\hline $\begin{array}{l}\text { Inflamatorio } \\
\text { (lesión cutánea, } \\
\text { artritis) }\end{array}$ & & $\begin{array}{l}\text { Lesiones de tejidos e } \\
\text { inflamaciones }\end{array}$ & $\begin{array}{l}\text { Dolor espontáneo } \\
\text { (pesado, difuso), } \\
\text { hipersensibilidad, } \\
\text { hiperalgesia y alodinia }\end{array}$ \\
\hline $\begin{array}{l}\text { Neurógeno } \\
\text { (neuralgia, lesión } \\
\text { espinal, lesión } \\
\text { talámica) }\end{array}$ & & $\begin{array}{l}\text { Lesión del sistema } \\
\text { nervioso central o } \\
\text { periférico }\end{array}$ & $\begin{array}{l}\text { Dolor espontáneo ('golpe } \\
\text { eléctrico,',cuchillazo') } \\
\text { Hiperalgesia y alodinia }\end{array}$ \\
\hline $\begin{array}{c}\text { Funcional } \\
\text { (fibromialgia, } \\
\text { síndrome talámico) }\end{array}$ & & $\begin{array}{l}\text { Hiperactivación o } \\
\text { pérdida de inhibición } \\
\text { de vías nociceptivas }\end{array}$ & $\begin{array}{l}\text { Dolor espontáneo, } \\
\text { difuso y profundo } \\
\text { Hiperalgesia y alodinia }\end{array}$ \\
\hline
\end{tabular}

Fuente: Elaboración propia a partir de Merchand (2009).

Este panorama de los tipos y mecanismos del dolor revela un dato fundamental del esquema científico del estudio del dolor, a saber: una estimulación no es indispensable para desencadenar un dolor. Lo anterior se explica, asimismo, por un dato bioanatómico: toda nocicepción tiene barreras inhibitorias a superar (Merchand 2009).

La primera barrera que toda transmisión del dolor debe sobrepasar es de carácter cognitivo: todo dolor debe sobreponerse a la barrera de la atención fijada por la consciencia (Melzack y Wall 1989).

Una segunda barrera es de carácter orgánico, la que se manifiesta en dos etapas del proceso de traspaso del dolor:

1. Modulación de la transducción nociceptiva. La estimulación debe mostrar una cierta intensidad para desencadenar sinapsis (+/- 42 grados Celsius o grados análogos de presión y/o acidez) y transmitirlas al sistema nervioso central.

2. Modulación de la transmisión nociceptiva. Cada etapa de la transmisión del dolor cuenta con sus propios mecanismos de modulación especializados. En este sentido, tanto las vías de transmisión ascendentes 
como descendentes tienen sus propios mecanismos de modulación del dolor. En el caso de las vías aferentes, se distinguen cuatro 'compuertas' de modulación del dolor (la entrada a la médula, el pasaje al bulbo raquídeo, el acceso al tronco cerebral y la llegada a la corteza cerebral). Las vías eferentes modulan el dolor gracias a la reducción de la actividad de neuronas nociceptivas no específicas (lo que implica que el dolor se desencadena a partir de la percepción cerebral de un contraste entre activación y excitación neuronal) (Merchand 2009).

Finalmente, existe una barrera psicológica del dolor. El sueño, la irritabilidad, el humor son estados que pueden disminuir el umbral del dolor, dependiendo de su coincidencia con la estimulación dolorosa (Melzack y Wall 1989).

Sin embargo, la enumeración de las barreras del dolor revela otra faceta del fenómeno del dolor en el enfoque científico, como lo es la existencia de un gradiente a la base de la experiencia dolorosa. La teoría de las compuertas propuesta por la investigación biológica para explicar la transmisión del dolor (Melzack y Wall 1989) pone al descubierto una analogía entre la estructura de la transmisión dolorosa y la experiencia personal del dolor. Desde un punto de vista subjetivo, el enfoque científico subraya que todo dolor puede comprenderse a partir del gradiente de Loeser: nocicepción-dolor-sufrimiento-expresión/comportamiento (Merchand 2009).

El gradiente de Loeser le permite al enfoque científico distinguir cuatro componentes en la experiencia dolorosa:

1. Componente nociceptivo. Todo dolor presupone la activación de fibras nociceptivas (pero esto no es suficiente para el desencadenamiento del dolor).

2. Componente sensodiscriminativo. Todo dolor supone la distinción entre sensación corriente y sensación dolorosa.

3. Componente afectivo. La diferenciación sensitiva no es suficiente para explicar el componente afectivo, ya que la vivencia de desagrado no se desencadena necesariamente como resultado del contraste sensorial.

4. Componente cognitivo-conductual. La sensación desagradable no implica la emergencia de expresiones o de conductas derivadas del dolor (rechazo y otras).

Luego de esta reconstrucción, pareciera que el enfoque científico del estudio del dolor representa una posición sólida y, sobre todo, ex- 
haustiva a la hora de dar cuenta de la realidad diferencial del fenómeno del dolor: se trata de un enfoque que pareciera agotar el fenómeno del dolor desde los prerrequisitos de su surgimiento hasta el linde que señala el sufrimiento. Sin mediar lo anterior, mostraremos en el próximo apartado las contradicciones, límites y supuestos inconfesados del enfoque científico del estudio del dolor.

\section{Límites epistemológicos del enfoque científico del estudio del dolor}

Antes de embarcarnos en una problematización de los presupuestos y operaciones analíticas del enfoque científico del estudio del dolor, es preciso realizar una caracterización epistemológica de sus rasgos analíticos y una explicitación de sus premisas teóricas. El hecho de que el enfoque científico del estudio del dolor encuentre su lugar dentro de los límites de la ciencia positiva moderna, determina el tratamiento de toda aproximación biológico-científica del dolor:

1. Dado que todo proceso anatómico y todo fenómeno estesiológico son considerados como una objetivación de una ley científica, el sujeto reconoce la integralidad de su experiencia sensitiva en el desenvolvimiento de la legalidad epistemológica. Todo dolor no es más que una concreción de reglas inherentes a la naturaleza. Este enfoque es intelectualista (en tanto interpreta como rasgos diferenciales de la experiencia cotidiana, resultados que forman parte del trato contingente del espíritu humano con la esfera de la ciencia).

2. Todo dato - toda hylé- del dolor nos reconduce a un proceso supuestamente más básico, más fundamental. Toda sensación disuelta en la cinemática y toda química diluida en la sinapsis, vuelven posible y legítimo delimitar —o, más bien, descomponer - intelectualmente el dolor real. El enfoque científico del estudio del dolor se revela, así, como analítico: en el esquema abstracto que él elabora no aparece la integridad del dolor padecido.

3. En el marco de esta descomposición radical del dolor, la sensación es movimiento, el movimiento es roce, el roce es reacción química y la reacción química es sinapsis (secuencia a la que se llega por la necesidad de leyes naturales). El enfoque científico del dolor es, asimismo, atomista: cada capa explicativa es reducida a un estrato más sólido (y supuestamente de mayor rendimiento cognitivo). 
4. Se deriva de este enfoque del dolor una oscilación entre la apuesta de describir rigurosamente el dolor (de ahí la referencia a la idea de 'objetividad'), y la pseudojustificación de fundamentar la investigación científica en la operatividad del sistema de expertos y en la idea de 'dato' o 'hecho'. Se trata, entonces, de un enfoque fáctico-pragmático: él disimula su debilidad epistemológica apelando a una noción ambigua de objetividad, al 'peso de los hechos' y a la irreversibilidad del 'estado de cosas' de la investigación científica.

El panorama de los rasgos epistemológicos diferenciales del enfoque científico del estudio del dolor nos redirige a una discusión sobre los límites explicativos y temáticos de toda tentativa de describir científicamente el dolor. En este sentido, haremos uso de la metodología de crítica de las ciencias parciales desarrollada por Husserl (2015) en sus Prolegómenos a la lógica pura - y llevada al ámbito de la fenomenología del dolor por diversos especialistas en la materia (Geniusas 2013; Morris 2013; Scarry 1985; Serrano de Haro 2013). En tanto la fenomenología busca conseguir una descripción de la experiencia pura del dolor que tenga como resultado un concepto claro y riguroso del fenómeno en cuestión, la metodología crítica de Husserl - y de toda la fenomenología - a la hora de examinar el proceder de las ciencias parciales, es la reducción al absurdo y el examen de rigurosidad de las conclusiones epistemológicas. A continuación se expondrán las contradicciones y limitaciones lógicas y epistemológicas del enfoque científico del estudio del dolor:

a. Limitación paradigmática. En lo relativo a la consistencia cognitiva del enfoque científico del estudio del dolor, se detecta una contradicción interna. Aun cuando el enfoque científico del estudio del dolor confía en el aporte explicativo de las leyes de la naturaleza (particularmente la teoría de las sinapsis) para demostrar cuál es el acicate capital del dolor, la tradición científica requiere de una referencia empírica para asociarla a la sensación dolorosa. De ahí la tendencia del enfoque científico de atribuir el acontecimiento puntual del dolor a la existencia de un tejido dañado u órgano afectado (Geniusas 2013). No obstante lo anterior, la necesidad de conceptos como 'tejido dañado' u 'órgano afectado' por parte del enfoque científico revela una contradicción interna en la aproximación científica del dolor, al punto de traicionar su propia lógica: por mucho que la materia esté sujeta a leyes físicas, el enfoque científico se 
ve obligado a atribuir el desencadenamiento del dolor a un concepto subordinado a su propia base teórica.

b. Limitación epistemológica. En cuanto al rigor de su criterio de validez, el enfoque científico del estudio del dolor exhibe una posición epistemológica débil. Aunque el criterio para determinar el desencadenamiento del dolor reside en la materia (herida, daño físico) o en la teoría de las sinapsis, ambos elementos son epistemológicamente débiles en comparación con la certeza de la experiencia subjetiva del dolor. Un tejido puede, por cierto, estar deteriorado sin dolor $-\mathrm{y}$ una sinapsis puede desencadenarse sin dolor-, pero la autoevidencia de la sensación íntima del dolor es irrebatible (Serrano de Haro 2013).

c. Limitación sistemática. En términos de su alcance explicativo, el enfoque científico del estudio del dolor muestra, asimismo, limitaciones considerables. En tanto este enfoque toma la forma de un análisis externo, se trata necesariamente de una descripción del dolor en tercera persona: ella pasa por alto la experiencia personal, la vivencia íntima del dolor (Geniusas 2013).

d. Limitación temática. Las limitaciones del enfoque científico del estudio del dolor son consecuencia de sus límites teóricos. Dado que este enfoque se delimita a partir del examen de los desencadenantes biofísicos del dolor, él no alcanza a explicar rigurosamente los dolores que no poseen acicates empíricos de tipo causal. De aquí que el enfoque científico no pueda ofrecer una explicación sólida de la relación entre dolor agudo y dolor crónico (ni de la naturaleza específica de este último) (Morris 2013).

e. Limitación programática. Finalmente, el enfoque científico se revela como poco operativo para captar el dolor, en la medida en que sus rasgos y elecciones epistemológicas fundamentales (intelectualismo, análisis, atomismo, pragmatismo) lo condenan a ser un enfoque de tipo estático (mientras que el dolor es un fenómeno esencialmente dinámico: todo dolor se caracteriza por una evolución temporal interna del padecer) (Scarry 1985).

Una vez explicitados los límites epistemológicos y las inconsistencias argumentativas del enfoque científico del estudio del dolor, estamos en condiciones de afirmar que él se revela como un paradigma intelectual que no muestra toda la rigurosidad deseable a la hora de captar el fenómeno del dolor. En el próximo apartado veremos cómo la fenomenología propone hacerse cargo de las mencionadas insuficiencias analíticas. 


\section{La fenomenología como superación de los límites del enfoque científico}

La reconstrucción del argumento central del enfoque científico del estudio del dolor y de sus limitaciones epistemológicas nos ha permitido llegar a la siguiente conclusión: el enfoque científico del estudio del dolor se muestra - a pesar de sus muchos méritos expuestos en la introducción de nuestro artículo- como una perspectiva epistemológica sin la rigurosidad necesaria para captar el fenómeno del dolor en su totalidad diferencial.

En ese sentido, la descripción fenomenológica del dolor se revela como una posibilidad única para sobreponerse a las inconsistencias analíticas del enfoque científico del estudio del dolor. No es casualidad: la filosofía fenomenológica nace específicamente para superar la debilidad epistemológica derivada de la primacía descriptiva de las ciencias parciales (Husserl 1950, §2) y no solo en nombre de su falta de rigurosidad descriptiva (Housset 2000, 26). Toda aproximación conceptual abandonada a los presupuestos irreflexivos propios de la descripción de las ciencias positivas tiene como destino impajaritable encontrarse, no con el fenómeno supuestamente descrito, sino con una deformación o espejismo resultantes de la falta de revisión de los propios presupuestos intelectuales (Husserl 1950, §8). Ahí se encuentra, justamente, la ironía de la descripción positiva del enfoque científico del estudio del dolor: en su tentativa de descripción 'pura' del dolor, él no es consciente de que su semántica y su terminología conllevan ya una carga intelectual (positivismo, mecanicismo, empirismo) que resulta en una descripción intelectualizada -y no pura - del fenómeno del dolor (Buytendijk 2001, 269).

El primer movimiento intelectual de la fenomenología a la hora de captar la especificidad diferencial del fenómeno del dolor será, entonces, luchar contra el vicio del propio pensamiento, a saber: el intelectualismo, el espejismo del conceptualismo; el solipsismo como consecuencia de una descripción irreflexivamente intelectualizada. Es por esto que el primer paso de una fenomenología del dolor es proceder a una desintelectualización del fenómeno del dolor (Buytendijk 1965, 125-142).

¿Cómo es posible desintelectualizar el intento descriptivo del fenómeno del dolor? E. Husserl (1950, 1998, 2004) ofreció una respuesta a esta misma pregunta, pero para todo intento descriptivo posible de 
cualquier ámbito de la realidad. La respuesta de Husserl no deja lugar a dudas, a saber: la experiencia. Este es el sentido de la máxima fenomenológica 'volver a las cosas mismas': la experiencia original del mundo cotidiano es la fuente de derecho de todo conocimiento y de todo saber (Husserl 1950, §24). No debe restringirse empero este concepto de experiencia a la utilización del mismo en el empirismo inglés (Romano 2010, 469-480): la experiencia es la disposición fundamental para el establecimiento de la relación entre consciencia y mundo, en tanto ella no solo nos ofrece el objeto a experimentar, sino un horizonte para el despliegue de la experiencia en particular, en general (y para nuevas referencias y apariciones experienciales) (Gadamer 1993, 430).

El primer paso fundamental para una descripción rigurosa del fenómeno del dolor lo constituye, entonces, apostar por una descripción de la experiencia subjetiva del dolor padecido, de la evidencia antepredicativa que solamente la experiencia cotidiana del dolor entrega (De Waelhens 1950).

Pero la tarea de captar el fenómeno del dolor en la experiencia de su padecer originario requiere, por cierto, de una determinada disposición intelectual. La filosofía fenomenológica propone una clave metodológica para satisfacer los requisitos analíticos que un abordaje conceptual del dolor exige. La fenomenología muestra que no se trata de definir las herramientas teóricas de manera abstracta ni de sistematizarlas a priori a la hora de captar el dolor. Por el contrario, es el dolor en su fenomenalización misma el que entrega autónomamente los elementos que vuelven posible su descripción filosófica (De Waelhens 1953). No por casualidad escribirá M. Henry $(2001, \S 12)$ que el principio último de la fenomenología es: "tanto aparecer, tanto ser".

Se trata, así, de atenerse a los rasgos diferenciales e invariantes de la experiencia del dolor en su consistencia característica. Dicho de otra forma, la tarea de una descripción rigurosa del fenómeno del dolor comienza necesariamente con la descripción de las formas y variantes esenciales con las que el dolor se da a la consciencia. Husserl (1950, §36) subraya que, no solo el dolor, sino todo fenómeno de cualquier tipo aparece ante la consciencia como consecuencia de la operación capital de toda consciencia, como lo es su naturaleza intencional:

Lo que caracteriza a la intencionalidad es la unidad de una acción de la consciencia con lo que se produce en ella [...] el 'ver' y lo 'visto' no pueden 
separarse el uno del otro [...] Del mismo modo, podemos decir: 'Yo he deseado (he cumplido un acto de deseo), por eso deseo' [...] La acción de la consciencia es intentio, es decir: el producto está contenido en el acto productivo. (Szilasi 1973, 40-41; cursivas y comillas del autor)

Llegamos así a una primera contribución de la fenomenología en su intento de captar rigurosamente la consistencia diferencial del fenómeno del dolor, a saber, el dolor es inseparable de la consciencia que lo percibe: “Comprendamos bien que el dolor 'de estómago' es el estómago mismo en tanto vivencia dolorosa [...] La gastralgia es el estómago presente a la consciencia como cualidad pura de dolor" (Sartre 2010, 396; mi traducción, comillas del autor).

Pero el principio fenomenológico de la intencionalidad de la consciencia no solo nos indica un camino metodológico para desintelectualizar la descripción de la experiencia del dolor en la que cayó el paradigma científico, sino que él permite, asimismo, resolver la cuestión de la problemática descripción en tercera persona del fenómeno del dolor llevado a cabo por el enfoque científico.

La descripción husserliana de la consciencia como puerta de entrada a la experiencia originaria implica que la consciencia está ya-siempre involucrada y se ve interpelada (quiéralo o no) por el objeto experimentado: para la consciencia, el sentido de lo experimentado es ontológicamente inseparable del acto de experimentarlo (Landgrebe 2004). Esto da cuenta de que la estructura de la consciencia como acto de apertura a la experiencia tiene como condición de posibilidad una capacidad implícita de autoafección preintencional: "La consciencia no es nunca creación exnihilo, ella está siempre, más bien, motivada; ella remite a una sensibilidad que le da su impulso necesario" (Montavont 1999, 65; mi traducción).

En este sentido, una vez descubierta la capacidad dadora de experiencia propia de la consciencia intencional, Husserl mostrará que toda variante de todo acto intencional de cualquier tipo tiene como correlato ineludible una forma de afección pasiva de carácter preintencional:

El yo está siempre afectado [...] la afección del yo es obra de la pasividad, y esto en todos los niveles de la constitución. Al nivel de la esfera hylética inmanente, son las sensaciones, las pulsiones y los instintos que afectan al yo; al nivel de las esferas trascendentes, es la sedimentación de los objetos que el yo ha constituido en el transcurso de su génesis el factor afectante. (Montavont 1999, 72; mi traducción) 
Con justa razón afirma entonces E. Lévinas $(1994,75)$ que la gran revolución filosófica desde Husserl es que: "la sensibilidad es ya susceptible de razón". La fenomenología de Husserl es la primera corriente intelectual en tematizar y abordar filosóficamente la condición sintiente, afectante, padeciente propia de toda subjetividad (tanto en su dimensión estesiológica como emotiva) (Kocka 1980).

La explicitación del factor pasivo afectante propio del acto intencional - 'el fluir pasivamente originario' del sedimento experiencial de toda consciencia (Landgrebe 1965, 304; mi traducción)_-, nos conduce a la segunda gran contribución de la filosofía fenomenológica a la hora de abordar el fenómeno del dolor, a saber: toda descripción rigurosa del fenómeno del dolor exige dar cuenta de su dimensión autoafectante, de la integralidad del desenvolvimiento de la vivencia dolorosa en la resonancia de su padecer. No por casualidad supo escribir J. Millas (2009, 59-60):

¿Podría alguien pretender que mi dolor de muelas es el conjunto de movimientos que lo expresan y que lo que llama dolor mi consciencia es solo engañosa apariencia? Nadie, sin duda; el dolor no es dolor mientras no lo padece por dentro una consciencia. Como desde fuera no puede sufrirlo espectador alguno, su realidad íntegra [...], su cualidad de dolor, se agota en la fase subjetiva.

La reconstrucción de los principios analíticos de la filosofía fenomenológica — regreso a la experiencia originaria, intencionalidad y pasividad autoafectante de la consciencia - indica un camino para sobreponerse, no solo a los límites descriptivos del enfoque científico del estudio del dolor (intelectualismo, descripción en tercera persona), sino para avanzar hacia una descripción rigurosa de la consistencia diferencial del fenómeno del dolor. Concluiremos este artículo esbozando una descripción fenomenológica del dolor que se hace cargo de los límites del enfoque científico.

\section{Elementos para una descripción fenomenológica del dolor}

En el apartado anterior establecimos, a partir de una reconstrucción de los presupuestos analíticos de la fenomenología, los requisitos que debe cumplir una descripción rigurosa del fenómeno del dolor. No existe mejor punto de partida para comenzar una descripción fundamental de la 
experiencia del dolor humano, que regresar al trabajo más decisivo alguna vez escrito sobre filosofía del dolor. Estamos pensando, por cierto, en el texto del fenomenólogo holandés F. Buytendijk (1965), Teoría del dolor.

La tesis fundamental de Buytendijk $(1965,133)$ es que el dolor es una amenaza no destructiva para el ego. Es justamente en virtud de la operatividad diferencial del fenómeno del dolor que se explica, a su vez, la reacción humana privativa frente a él, a saber: el estado de alienación. En este sentido, Buytendijk $(1965,173)$ muestra que, en la experiencia del dolor, el hombre no siente su cuerpo como lo hace habitualmente: el dolor está en mí, pero no forma parte de mí. Esta alienación intracorporal se expresa a través de la manifestación fenomenológicamente más característica del dolor, como lo es la localización de la sensación dolorosa. A diferencia de todos los demás tipos de padecer posibles (sufrimiento, asco, cansancio, miedo, aturdimiento, etcétera), el dolor se distingue fenomenológicamente por la localización corporal o concentración estesiológica del malestar. Pero hay más: Buytendijk (1965) considera que es precisamente gracias al fenómeno de la localización que el hombre puede tomar consciencia de su dolor. La amenaza del dolor se revela como no destructiva en tanto ella aparece restringida a una zona del cuerpo (por lo que ella no apunta a la totalidad de la integridad subjetiva (Buytendijk 1965, 145).

Sin embargo, debe destacarse que la sensación de alienación desencadenada por la amenaza no destructiva del dolor introduce una serie de cambios a nivel del cuerpo vivido (Tatossian 1982). La primera modificación característica del dolor en el ámbito corporal es la fijación del horizonte de movimiento: en todo dolor, el horizonte del movimiento se subordina a las posibilidades de desplazamiento prescritas por la zona adolorida (Leder 1990). De aquí que el dolor desencadene un movimiento centrípeto, vale decir, que el dolor contrae la disposición espacial del cuerpo hacia la subjetividad: en la experiencia del dolor, el cuerpo se aleja espontáneamente del mundo para refugiarse en sí mismo (Leder 1992).

El dolor tiene como consecuencia, asimismo, el desencadenamiento de un movimiento centrífugo: la vivencia del dolor obliga al hombre a encontrar una nueva disposición hacia el mundo para tolerar o para sobreponerse a él (Leder 1985, 256). Todo dolor implica una acción como reacción frente a la sensación desagradable: puede tratarse aquí de un 
movimiento deliberado (elongación) o de un reflejo de acomodación, es decir, de un movimiento que apunta a encontrar una nueva disposición corporal para hacer frente al dolor (como sucede en el caso del dolor crónico) (Leder 1990).

Al mismo tiempo, el dolor implica modificaciones en el ámbito de la temporalidad del cuerpo vivido (Leder 1985, 257). La experiencia del dolor fija el tiempo en el presente: durante el dolor no experimentamos más que el instante doloroso, la temporalidad intropática en la vivencia del dolor parece suspendida a tal punto que nuestro horizonte temporal no puede sobreponerse a la sensación dolorosa y nuestra memoria no tiene otro contenido que el momento de la lesión. No por casualidad escribirá el psiquiatra alemán T. Fuchs (2003, 70; mi traducción): "el dolor es la inevitable experiencia del ahora".

No obstante lo anterior -y como resultado de la fijación en el presente-, el individuo intenta evadirse a través de la nostalgia del estado predoloroso (la consciencia del sujeto adolorido escapa así hacia el pasado). Asimismo - y al mismo tiempo-, el dolor gatilla una proyección de la consciencia individual hacia el futuro, buscando refugio en la esperanza del alivio (Leder 1985, 258).

Cabe destacar que los cambios del dolor modifican también la forma de aparición del cuerpo vivido. El psiquiatra norteamericano D. Leder (1990) ha mostrado de manera admirable que el cuerpo vivido se distingue específicamente del cuerpo orgánico gracias a su capacidad de desaparecer. Las dos grandes formas de manifestación del cuerpo vivido tienen como precondición la aparición mediante la desaparición: el cuerpo vivido puede desenvolverse como sedimento de motivaciones sensokinestésicos solamente dejando esta función en la esfera de lo tácito, mientras que la manifestación del cuerpo vivido en tanto herramienta práctico-disposicional se vuelve posible exclusivamente manteniendo esta condición bajo el silencio de la virtualidad operativa (Leder 1990).

Pero, ¿cómo altera específicamente el dolor la aparición del cuerpo vivido? La modificación de la aparición del cuerpo vivido durante el dolor se lleva a cabo mediante dos movimientos. En primer lugar, el dolor concentra las motivaciones sensokinestésicas alrededor de la parte adolorida y, volviendo explícita la condición de sedimento sensorial del cuerpo vivido, el dolor hace aparecer a la corporeidad como dependiente de la hipersensibilización de una región orgánica del cuerpo (Leder 1990). 
A su vez, el dolor impide la aparición del cuerpo vivido como herramienta práctico-disposicional, dado que el dolor desata un movimiento de coerción cinestésico inmanente: el mundo deja de ser la referencia de la disposición corporal y el cuerpo vivido se dispone como consecuencia de la opresión física que el dolor desencadena. Es a causa de estos dos movimientos en el ámbito de la corporeidad que toda vivencia de dolor conlleva un llamado hermenéutico: las nuevas formas de aparición del cuerpo vivido que derivan del dolor crean la necesidad de una explicitación con respecto a dichos cambios (Leder 1990).

Podemos ver que la descripción fenomenológica del dolor se ha concentrado en la descripción intencional de la alienación resultante de la amenaza no destructiva que el dolor implica para el ego (así como de sus expresiones en la esfera del cuerpo vivido). No obstante, como vimos en el apartado anterior, todo estado de alienación supone una proyección personal o resonancia subjetiva de carácter no-intencional.

Es en esta dualidad -alienación corporal como resonancia subjetiva- donde la fenomenología encuentra el segundo aspecto esencial del dolor humano. El fenómeno del dolor, al mismo tiempo (y a pesar de la alineación), implica siempre un sentimiento de autoidentificación con la sensación de malestar anclada a la experiencia del dolor:

\footnotetext{
Nuestros dolores, que son una de las cosas que se encuentran en el mundo de cada cual o subjetivo, tienen una dimensión positiva en virtud de la cual sentimos por ellos algo así como un afecto - al mismo tiempo que nos están exasperando-, esa como difusa pero cálida actitud que sentimos hacia todo lo auténticamente nuestro. $Y$ es que mientras nos duele nos está, en efecto, siendo íntimo. ¿Cómo no va a ser así, si en el dolor soy siempre yo quien me duelo a mí mismo? (Ortega y Gasset 1964, 175-176; cursivas del autor)
}

En este punto de nuestro artículo podría parecer que nuestra tarea ha llegado a su fin, en tanto las herramientas descriptivas de la fenomenología nos han permitido sobreponernos a las limitaciones capitales del enfoque científico a la hora de describir el dolor. No obstante, la descripción fenomenológica de la vivencia del dolor supone una contribución adicional a la mera descripción de los rasgos esenciales del dolor. El encuentro con la experiencia originaria del dolor derivado de la descripción fundamental del fenómeno del dolor, nos conduce a la pregunta 
filosófica por antonomasia, a la pregunta por la significación esencial de la experiencia del dolor en cuanto tal.

En este sentido, la fenomenología muestra que el malestar inherente a la vivencia del dolor se construye a partir de la conjunción e íntima convivencia de alienación y autoidentificación. ¿Cuál es, entonces, el sentido de esta particular conjunción?

Desde un punto de vista teórico, la única experiencia que fusiona los opuestos -alienación y autoidentificación- es la experiencia del límite. En el límite estoy 'aquí' $y$, al mismo tiempo, estoy 'más allá', ya que, como escribiera K. Jaspers (1986, 423; mi traducción): "la palabra límite significa [...] ser aún inmanencia e indicar ya la trascendencia".

La experiencia de la que habla Jaspers muestra exactamente el sentido diferencial del dolor: la autoidentificación en el dolor da cuenta de la parte más propia de mi mismidad (del 'aquí y ahora de mi cuerpo'), mientras que la alienación nos habla del estado de virtualidad en el que mi cuerpo se encuentra ya más allá de su inmediatez. Esta dualidad pone de relieve el significado fundamental del fenómeno del dolor, a saber: el sentido del dolor es la experiencia del límite de mi propio cuerpo (Mujica 2018). En cada dolor llevamos a cabo la experiencia del límite de nuestro cuerpo, es decir, alcanzamos los límites que nuestro esquema corporal ha sistematizado como respuesta a los estímulos sensokinestésicos, a la vez que nos encontramos con los límites de nuestro'Yo-puedo' (MerleauPonty 2003) y de nuestra capacidad de resistencia frente al mundo (Mujica 2018). Los individuos que padecen insensibilidad congénita al dolor grafican perfectamente el sentido ínclito de la experiencia del dolor: se trata de cuerpos que parecieran no tener resistencia, de cuerpos sin delimitación estesiológica, de una corporeidad sin límites.

La explicitación de las herramientas diferenciales de la fenomenología no solo nos permitió acceder a matices del fenómeno del dolor a los que no podía acceder el enfoque científico, sino que, más aún, la descripción esencial de la vivencia del dolor nos condujo a la determinación del sentido integral de la experiencia del dolor. A modo de conclusión, mostraremos cuál es el rendimiento de contar con un concepto claro y riguroso de la experiencia dolorosa (concepto específicamente posibilitado por la descripción fenomenológica y sus instrumentos teóricos característicos). 


\section{Conclusión. Sobre las prestaciones de una fenomenología del dolor}

La reconstrucción esquemática de los presupuestos fenomenológicos del tratamiento del dolor (alienación, autoidentificación, límite del propio cuerpo) nos ha permitido, no solo acceder al sentido de la experiencia del dolor, sino a sopesar nuevamente la contribución filosófica de la fenomenología en su abordaje de la experiencia dolorosa. De esta forma, la filosofía fenomenológica no solo contribuye con una descripción esencial de la vivencia del dolor, sino que nos permite contar con un concepto riguroso del dolor mismo, ya que, como escribiera Buytendijk (2001, 269; mi traducción): "Es imposible comprender o incluso describir las relaciones sexuales cuando se ignora la esencia y la significación de la sexualidad, de la existencia corporal, de la feminidad y de la masculinidad".

En este sentido, la tentativa de describir fenomenológicamente el dolor conlleva como prestación avances que sobrepasan el ámbito estrictamente filosófico. Desde esta óptica, la validez del método fenomenológico a la hora de abordar la experiencia del dolor reside en su compromiso irredimible con el acrecentamiento del rigor epistemológico de las esferas de investigación. No por casualidad considera Merleau-Ponty (2001) que la fenomenología conserva hasta nuestros días el espíritu epistemologizante que Husserl le imprimió. No obstante, este incremento epistemológico característico del proyecto de la fenomenología (Merleau-Ponty 2001) no encuentra su razón en un afán intelectualista. La búsqueda de claridad y rigor por parte de la fenomenología reside en que, una filosofía rigurosamente fundada, implica un aumento del rendimiento del pensamiento y, por lo tanto, de la ciencia, de la técnica y, en último término, de la tesitura y sedimento de la cultura humana misma (Husserl 1990). Este argumento puede aplicarse mutatis mutandi a la experiencia del dolor, a saber: solo a partir de fundamentos analíticos rigurosos y de una conceptualización clara de la vivencia del dolor, puede derivarse una comprensión profunda de la significación del padecer del paciente — de sus matices y su extensión-, así como de la eficacia de las posibles intervenciones, sus variantes y las herramientas pertinentes para hacer frente a la experiencia del dolor en toda su profundidad, variabilidad y riqueza.

En el contexto de este artículo, el argumento fenomenológico permite mostrar que un enfoque del dolor llevado a cabo a partir de una 
base intelectual sólida — como lo es una descripción fundamental de la experiencia del dolor-, tiene como consecuencias:

1. Claridad en torno a la delimitación del objetivo fundamental de las intervenciones prácticas. En este sentido, una fenomenología del dolor puede resultar decisiva a la hora de circunscribir los procedimientos y las estrategias terapéuticas para enfrentar el dolor. Esto puede resultar en un incremento diferencial de la eficacia de las intervenciones prácticas frente al dolor.

2. Claridad sobre el alcance de las intervenciones prácticas. De esta forma, una descripción fenomenológica del dolor ayuda a delimitar el marco, el rendimiento y la eficacia de los procedimientos para abordar terapéuticamente el dolor. Esto puede representar el fundamento de una metodología para evaluar y diseñar las intervenciones terapéuticas frente al dolor.

3. Claridad con respecto a las condiciones de posibilidad del dolor. En esta óptica, una fenomenología del dolor se revela como una herramienta capital para superar las nociones positivistas del cuerpo humano. Esta elucidación tendría como consecuencia directa la reconsideración científica de fenómenos como la asimbolia dolorosa, el dolor irradiado, el miembro fantasma, la alodinia o el dolor volumétrico (Grahek 2007). Asimismo, esta contribución puede resultar fundamental a la hora de evaluar la eficacia y la atingencia terapéutica de los modelos 'causa-efecto' a la hora de curar o aliviar el dolor.

4. Claridad sobre la naturaleza diferencial del dolor y sobre la especificidad de sus variantes. Una descripción fenomenológica del dolor permite abandonar la visión estática con la que el enfoque científico ha caracterizado la experiencia dolorosa. Lo anterior permitiría dilucidar mucho más claramente el desenvolvimiento de la experiencia del dolor, lo que tendría como consecuencia una mejor comprensión terapéutica de la relación entre dolor agudo y dolor crónico (así como una distinción mucho más nítida entre dolor y sufrimiento) (Ricoeur 1992). Lo anterior constituye un camino para desarrollos terapéuticos centrados en el dolor crónico.

Pero la contribución más decisiva de una fenomenología del dolor remite al espíritu filosófico que ella conlleva. Contrariamente a las ciencias positivas - las que generan soluciones específicas para problemas parciales-, la filosofía es un esfuerzo de estructuración del mundo a 
partir de una idea que entregue una respuesta universal a los conflictos de nuestra circunstancia histórica. En este sentido, una descripción rigorosa sobre los rasgos característicos, la consistencia diferencial y el sentido esencial del fenómeno del dolor, constituye un paso único para comprender el rol que la experiencia del dolor juega en la vida del hombre.

\section{Bibliografía}

Boutot, A. 1995. La pensé allemande moderne. Paris: PUF.

Buytendijk, F. 1965. Teoría del dolor. Buenos Aires: Troquel.

Buytendijk, F. 2001. Approche phénoménologique du problème des sentiments et des émotions. Alter 9, 251-270.

De Waelhens, A. 1950. La phénoménologie du corps. Revue Philosophique de Louvain. Tercera serie, tomo 48(19), 371-397.

De Waelhens, A. 1953. Phénoménologie et vérité. Essai sur l'évolution de l'idée de vérité chez Husserl et Heidegger. Paris: PUF.

Fuchs, T. 2003. The Temporality of Pain and Suffering (69-75). En Granger, B. y Charbonneau, G. (eds.), Phénoménologie des sentiments corporels I. Douleur, souffrance, dépression. Paris: Cercle Herméneutique.

Gadamer, H.G. 1993. Verdad y método. Salamanca: Sígueme.

Geniusas, S. 2013. On Naturalism in Pain Research: A Phenomenological Critique. Metodo: International Studies in Phenomenology and Philosophy 1(1), 1-10.

Grahek, N. 2007. Feeling in Pain and Being in Pain. Boston: MIT Press.

Henry, M. 2001. Incarnation. Une philosophie de la chair. Paris: Seuil.

Henry, M. 2011. L'essence de la manifestation. Paris: PUF.

Housset, E. 2000. Husserl et l'énigme du monde. Paris: Points.

Husserl, E. 1950. Idées directrices pour une phénoménologie. Paris: Gallimard.

Husserl, E. 1990. La crisis de las ciencias europeas y la fenomenología trascendental. Barcelona: Crítica.

Husserl, E. 1998. De la synthèse passive. Grenoble: Jérôme Millon.

Husserl, E. 2004. Idées directrices pour une phénoménologie et une philosophique phénoménologiques pures 2. Recherches phénoménologiques pour la constitution. Paris: PUF.

Husserl, E. 2015. Investigaciones lógicas 1. Madrid: Alianza.

Jaspers, K. 1986. Philosophie: orientation dans le monde, éclairement de l'existence, métaphysique. Paris: Springer-Verlag.

Kandel, E., Schwartz, J. y Jessell, T. 2001. Principios de neurociencia. México DF: McGraw Hill.

Kocka, U. 1980. Phänomenologische Konstitution und Lebenswelt. Untersuchungen zu Edmund Husserls Ideen II. Bielefeld: Kleine Verlag.

Landgrebe, L. 1965. Die Phänomenologie der Leiblichkeit und das Problem der Materie (291-306). En Landgrebe, L. (ed.), Beispiele. Fetschrift für Eugen Fink zum Geburstag. La Haya: Martinus Nijhoff.

Landgrebe, L. 2004. Le problème de la constitution passive. Alter 12, 235-248.

Leder, D. 1985. Towards a Phenomenology of Pain. Review of Existential Psychology and Psychiatry 19, 255-266. 
Leder, D. 1990. The Absent Body. Chicago: Chicago University Press.

Leder, D. 1992. The Experience of Pain and its Clinical Implications (83-97). En Peset, J. y Guillen, D. (eds.), The Ethics of Diagnosis. Cham: Springer.

Lévinas, E. 1968. Reflexiones sobre la 'técnica' fenomenológica (88-100). En De Maci, G. (ed.), Husserl: Tercer Coloquio Filosófico de Royaumont. Buenos Aires: Paidós.

Lévinas, E.1994. Théorie de l'intuition dans la phénoménologie de Husserl. Paris: Vrin.

Melzack, R. y Wall, P. 1989. Le défi de la douleur. Paris: Vigot.

Merchand, S. 2009. Le phénomène de la douleur. Comprendre pour soigner. Paris: Elsevier Masson.

Merleau-Ponty, M. 2001. Phénoménologie de la perception. Paris: Gallimard.

Merleau-Ponty, M. 2003. L'institution dans I'histoire personnelle et publique; Le problème de la passivité: le sommeil, l'inconscient, la mémoire: notes de cours au collège de France, 1954-1955. Paris: Belin.

Millas, J. 2009. Idea de la individualidad. Santiago: UDP.

Montavont, A. 1999. De la passivité dans la phénoménologie de Husserl. Paris: PUF.

Morris, K. 2013. Chronic Pain in Phenomenological/Anthropological Perspective (167-175). En Jensen, R. y Moran, D. (eds.), The Phenomenology of Embodied Subjectivity. Contributions to Phenomenology. Cham: Springer.

Mujica, F. 2018. Éprouver la limite. Phénoménologie de la douleur. Tesis PhD, Université catholique de Louvain-La-Neuve.

Ortega y Gasset, J. 1964. El hombre y la gente (71-274). En Ortega y Gasset, J., Obras completas, tomo VII. Madrid: Revista de Occidente.

Ortega y Gasset, J. 1975. Sensación, construcción e intuición (109-126). En Ortega y Gasset, J., Apuntes sobre el pensamiento. Madrid: Revista de Occidente.

Purves, D. 1997. Neuroscience. Sunderland: Sinauer associates.

Ricouer, P. 1992. La souffrance n'est pas douleur. Psychiatrie française (Numéro spécial), $1-7$.

Romano, C. 2010. Au cœur de la raison: la phénoménologie. Paris: Gallimard.

Sartre, J.P. 2010. L'être et le néant. Essai d'ontologie phénoménologique. Paris: Gallimard.

Scarry, E. 1985. The Body in Pain. New York: Oxford University Press.

Serrano de Haro, A. 2013. El dolor de los marcianos. Un análisis fenomenológico contra Rorty. Investigaciones fenomenológicas. Volumen monográfico 4(I): Razón y vida, 313-330.

Spiegelberg, H. 1994. The Phenomenological Mouvement. A Historical Introduction. Boston: Kluwer Academic Publishers.

Szilasi, W. 1973. Introducción a la fenomenología de Husserl. Buenos Aires: Amorrortu.

Tatossian, A. 1982. Phénoménologie du corps (99-103). En Jeddi, E. (ed.), Le corps en psychiatrie. Colloque international Ibn Sina-Collomb. Paris: Elsevier Masson.

Wall, P. y Melzack, R. 1999. Textbook of Pain. London: Churchill Livingstone. EP 